Article

\title{
Synthesis of a Novel Benzoyl Adenosine Analog Containing a 1, 4-Dioxane Sugar Analog and the Synthesis of a Corresponding Uracil Adenine Dinucleotide
}

\author{
Qiang Yu and Per Carlsen* \\ Department of Chemistry, Norwegian University of Science and Technology, 7491 Trondheim, \\ Norway
}

* Author to whom correspondence should be addressed; E-Mail: per.carlsen@chem.ntnu.no; Tel.: +47-73593968.

Received: 3 March 2011; in revised form: 28 April 2011 / Accepted: 6 May 2011 /

Published: 12 May 2011

\begin{abstract}
Adenosine analogs in which the sugar unit was replaced by a 1,4-dioxane sugar equivalent, were prepared by coupling the 1,4-dioxane sugar analog as its anomeric acetates, with N6-benzoyl protected adenine. The 1,4-dioxane system was obtained in an enantioselective synthesis from $(R, R)$-dimethyl tartrate. Using standard phosphorimidite methodology, the adenine analog was further reacted with a 1,4-dioxane uridine analog to give the corresponding, protected dinucleotide, set-up for further condensation into larger oligonucleotides.
\end{abstract}

Keywords: nucleoside analogs; heterocyclic; adenosine; 1,4-dioxane; uracil-adenine

\section{Introduction}

Pursuing development of new antiviral and antitumor agents, a number of new nucleosides analogs have been synthesized in which of the sugar structures were modified [1]. The sugar unit has for example been replaced by a 1,4-dioxane moiety [2-6]. Some of these structures were reported to exhibit interesting biological activities [2,3], which may be ascribed to their particular flexible conformational properties [4]. Earlier we reported the synthesis of new optically active uridine analogs 1a or $\mathbf{1 b}$ where the sugar was substituted by an optically active 1,4-dioxane moiety [7]. These uridine analog was further elaborated into the corresponding dinucleotide using standard phosphorimidite 
methodology [8]. However, we also reported [7] that preparation of the related adenosine analog failed. In this communication we wish to report our recent findings dealing with the synthesis of adenosine analogs $\mathbf{2 a}$ and $\mathbf{2} \mathbf{b}$, which as the $N$-benzoyl protected compounds were then tested in dinucleotide formation with uridines $\mathbf{1 a}$.

Figure 1. Uridine and adenosine analogs containing a 1,4-dioxane sugar equivalent.<smiles>O=c1ccn(C2COC([C@@H](O)CO)CO2)c(=O)[nH]1</smiles>

1a: $\alpha$-anomer, trans-isomer

1b: $\beta$-anomer, cis-isomer<smiles>Nc1ncnc2c1ncn2C1CO[C@@H]([C@@H](O)CO)CO1</smiles>

2a: $\alpha$-anomer, trans-isomer

2b: $\beta$-anomer, cis-isomer

\section{Results and Discussion}

The synthesis of 1,4-dioxane sugar analog 3 from $(2 R, 3 R)$-dimethyl tartrate was reported earlier [7]. The tartrate was first converted into the corresponding enantiomerically pure diethyl $(2 R, 3 R)-2-O$ allyltartrate either by the reaction with allyl bromide in the presence of silver oxide or in a tin assisted reaction with dibutyltin oxide [9-11]. The allyl ether was reduced by $\mathrm{LiAlH}_{4}[12-14]$ or $\mathrm{NaBH}_{4}[15,16]$ to give a triol which was protected as an acetal with 2,2-dimethoxy-propane in the presence of $p$-toluene sulfonic acid. Subsequent ozonolysis afforded 1,4-dioxane sugar analog 3 which was further transformed into acetate 4 with acetic anhydride (Scheme 1). All the tartrate stereoisomers are readily available from the chiral pool, conveniently allowing for the synthesis of all the possible stereoisomers of the nucleoside analogs.

Scheme 1. Synthesis of 1,4-dioxane sugar analogs 3 and 4 [7].<smiles>COC(=O)C(O)C(O)C(=O)OC</smiles><smiles>[R]OC1COC(C2COC(C)(C)O2)CO1</smiles>

3: $\mathrm{RO}-=\mathrm{OH} ;$ cis + trans 4: $\mathrm{RO}-=-\mathrm{OAc} ;$ cis + trans

$i$ : allyl bromide (ref. 9-12)

ii. $\mathrm{LiAlH}_{4}$ (ref 12,14) or $\mathrm{NaBH}_{4}$ (ref 15)

iv. $\mathrm{O}_{3} / \mathrm{CH}_{2} \mathrm{Cl}_{2}$

iii. 2,2-Dimethoxypropane, $p \mathrm{TsOH}$

$v$, Acetic anhydride

\subsection{Synthesis of Adenosine Analogs}

The corresponding adenine nucleoside analog from $\mathbf{4}$ was attempted prepared using a Vorbrüggen procedure [17,18]. Thus, acetate 4 was coupled with silylated adenine 5 , in the presence of trimethylsilyl trifluoromethanesulfonate (TMSOTf) to give $\mathbf{6}$ as a cis/trans mixture in $23 \%$ yield after flash chromatography. However, HMBC-NMR spectroscopic analysis showed that the product was the 
undesired $\mathrm{N}-7$ regioisomer $\mathbf{6}$, as three bond correlations were observed for the anomeric proton with $\mathrm{C} 5$ as well as C8 (Scheme 2).

Scheme 2. Synthesis of N-7 adenine nucleoside analogs 6.<smiles>CC(=O)OC1COC([C@H]2COC(C)(C)O2)CO1</smiles>

4

5

6, trans and cis

For reasons of forcing the adenine to react at the 9-position, 4 was instead reacted with the silylated N6-benzoyl protected adenine, 7, in the presence of TMSOTf, affording the desired benzoyl protected adenine nucleoside analogues 8 as a cis/trans mixture in $42 \%$ isolated yield after chromatographic purification, Scheme 3. The structure was confirmed by NMR analysis.

Scheme 3. Synthesis of benzoyl adenine nucleoside analogues 8.<smiles>CC1(C)OCC(C2COC(OC(F)F)CO2)O1</smiles>

4<smiles>CS(C)(C)n1cnc2c(S(C)(=O)=O)ncnc21</smiles>

7<smiles>CC1(C)OCC(C2COC(n3cnc4c(NC(=O)c5ccccc5)ncnc43)CO2)O1</smiles>

8 trans and cis

Attempts to remove the acetal protection group in $\mathbf{8}$ failed, as depurination was observed to take place in all cases. A number of literature methods described were tested [19,20], such as cleavage using $80 \%$ acetic acid [21] or CSA as catalyst, reaction with Amberlyst 15, $\mathrm{HCl}$ [22] or trifluoroacetic acid [23]. Other reagents, such as ferric chloride on silica gel [24], iodine in methanol [25] or alumina or silica gel catalysis in all cases gave no or similar results. The desired benzoyl protected nucleoside analog 9 was finally obtained by the reaction sequence shown in Scheme 4.

Scheme 4. Synthesis of benzoyl protected adenine nucleoside analogue $\mathbf{9}$.

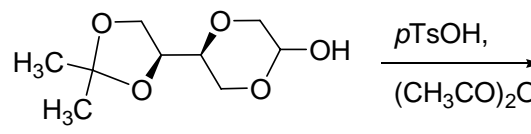

3

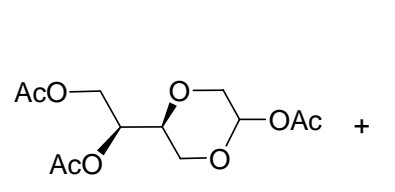

10: cis + trans

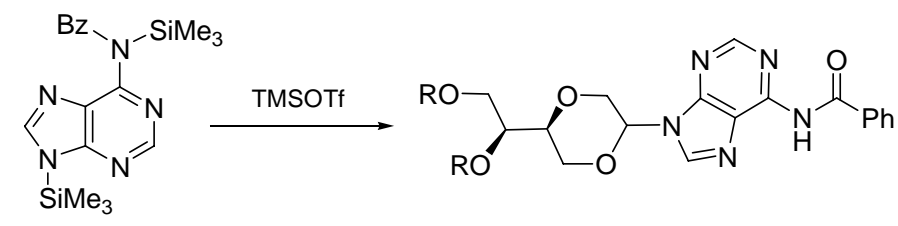

7

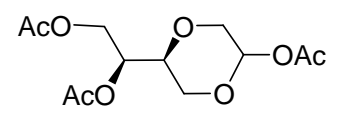

10: cis + trans 
Conversion of $\mathbf{3}$ to the corresponding triacetate $\mathbf{1 0}$ by the reaction with acetic anhydride in the presence of catalytic amount of $\mathrm{H}_{2} \mathrm{SO}_{4}$ [26,27] gave the desired product though in a poor yield. Product $\mathbf{1 0}$ was, however, obtained in a satisfactory yield when $\mathbf{3}$ were reacted with acetic anhydride in the presence of 1.1 equivalent of $p$-TsOH [28]. Subsequently the triacetate was smoothly coupled with the silylated benzoyl adenine 7 in the presence of TMSOTf to afford benzoyl adenine nucleoside analogue 11. The acetate groups were selectively removed by ammonia in methanol [29] leaving the desired, benzoyl-protected nucleoside analog 9 (Scheme 4).

\subsection{Synthesis of Dinucleotide Analogs}

The pure trans-nucleoside analog 9a was separated by flash chromatography from the mixture of diastereomers of compound 9. Treatment of 9a with 4,4'-dimethoxyltrityl chloride (DMTrCl) in pyridine [30] afforded the primary hydroxyl group protected compound 12a in 40\% yield after flash chromatography. Compound 12a was next reacted with $N, N$-diisopropyl-2-cyanoethylphosphor-amidic chloride [31,32] in the presence of $N, N$-diisopropylethylamine to give the desired phosphoramidite 13a in $51 \%$ isolated yield, Scheme 5.

Scheme 5. Synthesis of phosphoramidite 13a.

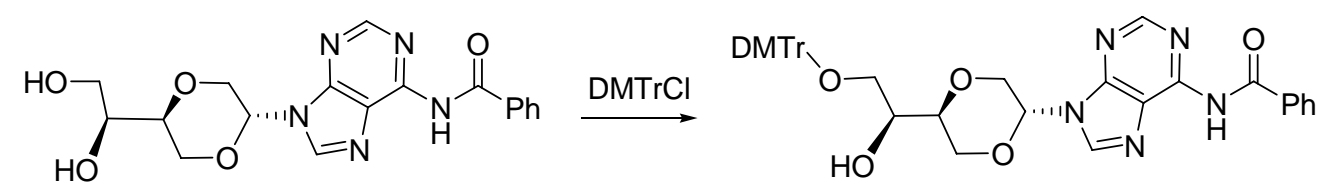

$9 a$

$12 \mathrm{a}$

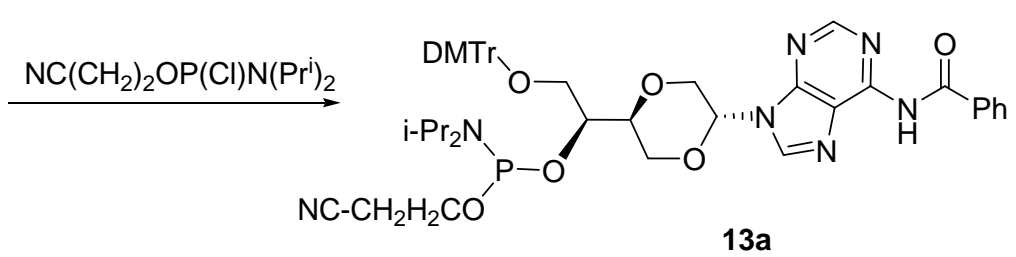

We have previously reported the synthesis of the uridine analog $\mathbf{1}$, obtained from acetates $\mathbf{4}$. The trans-anomer 1a was isolated in $42 \%$ yield after recrystallization from acetonitrile, and subsequently converted to the secondary acetate 14a [7].

Figure 2. Trans-uridine analogs 1a and 14a.<smiles>[R]O[C@H](CO)[C@H]1CO[C@@H](n2ccc(=O)[nH]c2=O)CO1</smiles>

1a: $\mathrm{R}=\mathrm{H}$

14a: $R=A c$ 
The coupling of adenosine phosphoramidite 13a with uridine analog 14a was carried out in the presence of $1 H$-tetrazole in dry acetonitrile [33], followed by iodine oxidation and purification by flash chromatography on silica gel. This afforded the uracil adenine dinucleotide analog $\mathbf{1 5}$ in $62 \%$ yield, Scheme 6 . To obtain the analytically pure dinucleotide 15 , the product was purified by multi elution preparative TLC.

Scheme 6. Synthesis of 1,4-dioxane uracil adenine dinucleotide analog 15.<smiles>CCOC(=O)C(CO)C1CO[C@@H](n2ccc(=O)[nH]c2=O)CO1</smiles>

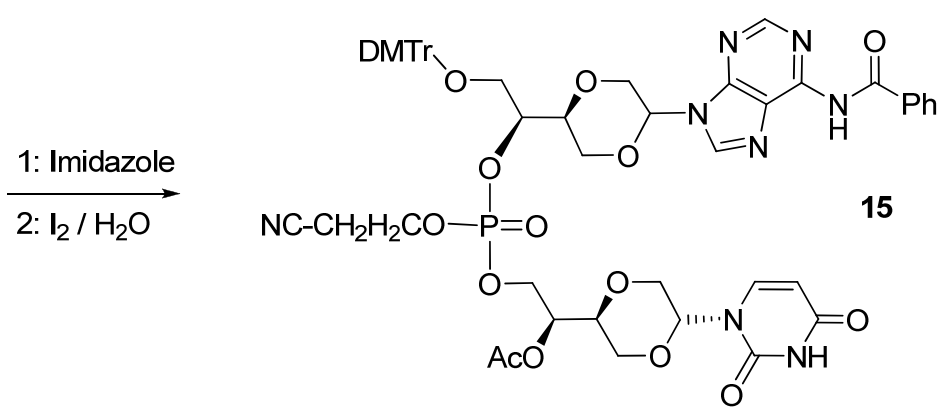

Further elaboration of product $\mathbf{1 5}$ was not pursued, as it at this stage was demonstrated that the 1,4-dioxane nucleoside analogs can function in the standard reaction scheme for oligonucleotide preparations, used in for example automatic nucleotide synthesis machines. Thus 15 represent a standard intermediate for the further preparation of oligonucleotides.

\section{Experimental}

\subsection{General}

NMR spectra were recorded on Bruker Avance DPX 300 or DPX 400 instruments. Chemical shifts are reported in ppm using TMS $(\mathrm{d}=0.0)$ as the internal standard in $\mathrm{CDCl}_{3}$ or relative to $2.50 \mathrm{ppm}$ for ${ }^{1} \mathrm{H}$ and $39.99 \mathrm{ppm}$ for ${ }^{13} \mathrm{C}$ in $\left[d_{6}\right.$-DMSO] or $3.31 \mathrm{ppm}$ for ${ }^{1} \mathrm{H}$ and $49.15 \mathrm{ppm}$ for ${ }^{13} \mathrm{C}$ in $\mathrm{CD}_{3} \mathrm{OD}$. Structural assignments were based on ${ }^{1} \mathrm{H},{ }^{13} \mathrm{C}$, DEPT135 and 2D-spetra, COSY, HSQC, HMBC, and NOESY. EI-Mass and ESI spectra were recorded on a Finnigan MAT 95XL spectrometer. IR spectra were obtained on a Thermo Nicolet FT-IR Nexus spectrometer using a Smart Endurance reflection cell. For ozonolysis was used an OZ-500 Ozone Generator produced by Fishcer Technology. Silica gel Kieselgel 60G (Merck) was used for Flash Chromatography. The solvents were purified by standard methods. All reactions were carried out in inert atmospheres (nitrogen). The synthesis of compounds 1a and 14a has been described elsewhere [7]. 
dry pyridine $(15 \mathrm{~mL})$ was added acetic anhydride $(0.62 \mathrm{~g}, 6 \mathrm{mmol})$ at $0-5{ }^{\circ} \mathrm{C}$ and the reaction mixture was stirred for 6 hours. The solution was concentrated under reduced in vacuo overnight, yielding the crude product in $87 \%$ yield as an oily solid material, which was used in the subsequent reaction step without further purification. The anomeric ratio 4a:4b (trans-cis ratio) was determined to be $4: 1$ by NMR. The products exhibited the following spectroscopic properties: The trans-product 4a (Figure 1) was assigned the following signals: ${ }^{1} \mathrm{H}-\mathrm{NMR}\left(\mathrm{CDCl}_{3}, 400 \mathrm{MHz}\right): \delta 1.36,1.43\left(\mathrm{~s}, 2 \times 3 \mathrm{H},\left(\mathrm{CH}_{3}\right)_{2} \mathrm{C}\right)$, $2.11\left(\mathrm{~s}, 3 \mathrm{H}, \mathrm{CH}_{3} \mathrm{COO}\right), 3.48\left(\mathrm{dd}, J=8.0 \mathrm{~Hz}, 11.4 \mathrm{~Hz}, 1 \mathrm{H}, \mathrm{H}_{\mathrm{B} 1}\right), 3.63\left(\mathrm{~m}, 1 \mathrm{H}, \mathrm{H}_{\mathrm{C}}\right), 3.69$ (dd, $\left.J=9.4 \mathrm{~Hz}, 11.4 \mathrm{~Hz}, 1 \mathrm{H}, \mathrm{H}_{\mathrm{D} 1}\right), 3.81\left(\mathrm{dd}, J=6.8 \mathrm{~Hz}, 8.0 \mathrm{~Hz}, 1 \mathrm{H},\left(\mathrm{CH}_{3}\right)_{2} \mathrm{C}-\mathrm{O}-\mathrm{CH}_{2}\right), 3.89$ (dd, $\left.J=2.6 \mathrm{~Hz}, 11.4 \mathrm{~Hz}, 1 \mathrm{H}, \mathrm{H}_{\mathrm{D} 2}\right), 3.93\left(\mathrm{dd}, J=2.8 \mathrm{~Hz}, 11.4 \mathrm{~Hz}, 1 \mathrm{H}, \mathrm{H}_{\mathrm{B} 2}\right), 4.00$ (dd, $J=6.8 \mathrm{~Hz}, 8.4 \mathrm{~Hz}$, $\left.1 \mathrm{H},\left(\mathrm{CH}_{3}\right)_{2} \mathrm{C}-\mathrm{O}-\mathrm{CH}_{2}\right), 4.15\left(\mathrm{~m}, 1 \mathrm{H},\left(\mathrm{CH}_{3}\right)_{2} \mathrm{C}-\mathrm{O}-\mathrm{CH}-\right), 5.74\left(\mathrm{dd}, J=2.8 \mathrm{~Hz}, 8.4 \mathrm{~Hz}, 1 \mathrm{H}, \mathrm{H}_{\mathrm{A}}\right) \mathrm{ppm}$. ${ }^{13} \mathrm{C}-\mathrm{NMR}\left(\mathrm{CDCl}_{3}, 100 \mathrm{MHz}\right): \delta 20.9,25.2,26.3,65.2,65.6,66.9,74.2,74.2,89.4,109.7,169.0$ ppm. The protons NMR spectrum of the corresponding cis- compound, $\mathbf{4} \mathbf{b}$, could not be fully assigned due to the peaks overlap with $\mathbf{4 a}$, however, the carbon NMR spectrum of the cis-compound was assigned the following signals: ${ }^{13} \mathrm{C}-\mathrm{NMR}\left(\mathrm{CDCl}_{3}, 100 \mathrm{MHz}\right): \delta 21.1,25.2,26.2,61.0,64.9,67.6,74.9,75.1$, 88.4, 109.7, $169.8 \mathrm{ppm}$. The mixture exhibited the following mass spectrum: MS (EI) $\mathrm{m} / \mathrm{z}: 247$ $\left(\mathrm{M}^{+}+1\right), 231\left(\mathrm{M}^{+}-\mathrm{CH}_{3}\right), 187\left(\mathrm{M}^{+}-\mathrm{OAc}\right), 145\left(\mathrm{C}_{6} \mathrm{H}_{9} \mathrm{O}_{4}\right)$. Elem. Anal. calcd. for $\mathrm{C}_{11} \mathrm{H}_{18} \mathrm{O}_{6}: \mathrm{C}$ 53.65, $\mathrm{H}$ 7.37; found C, 53.84, H 7.45.

Figure 1. Structure of compound 4a.

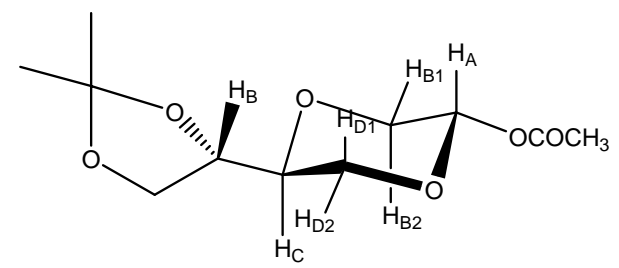

$4 \mathbf{a}$

$N-7-\{(2 R, 5 S)-5-[(4 S)-(2,2-D i m e t h y l-1,3-d i o x o l a n-4-y l)]-1,4-d i o x a n-2-y l\}$ adenine $\quad(6 a) \quad$ and $\quad N-7-$ $\{(2 S, 5 S)-5-[(4 S)-(2,2-d i m e t h y l-1,3-d i o x o l a n-4-y l)]-1,4-d i o x a n-2-y l\} a d e n i n e \quad(6 \mathbf{b})$. The mixture of adenine $(1.35 \mathrm{~g}, 10 \mathrm{mmol})$ and ammonium sulfate $(124 \mathrm{mg}, 0.9 \mathrm{mmol})$ in hexamethyldisilazane (HMDS, $35 \mathrm{~mL}$ ) was refluxed overnight. The solvent was evaporated and the residue was dissolved in dry dichloroethane $(20 \mathrm{~mL})$. To this solution, compound 4 (0.85 g, $3.5 \mathrm{mmol})$ was added. The solution was cooled to $0{ }^{\circ} \mathrm{C}$ and TMSOTf $(0.75 \mathrm{~mL}, 4 \mathrm{mmol})$ was added. The solution was stirred for 8 hours at room temperature. The chloroform $(50 \mathrm{~mL})$ was added and the solution was washed twice with saturated $\mathrm{NaHCO}_{3}$ solution $(15 \mathrm{~mL})$. The aqueous phase was extracted twice with chloroform $(50 \mathrm{~mL})$, and the combined organic layers was dried over anhydrous $\mathrm{MgSO}_{4}$ and filtered and concentrated under reduced pressure. The residue was purified by flash chromatography using a mixture of dichloromethane and methanol (19:1) as the eluent. A white solid (0.26 g, 23\%) was obtained which was identified as a 3:2 mixture of the trans- and cis- products $6 \mathbf{a}$ and $\mathbf{6 b}$. From the mixture of isomers was extracted the following spectroscopic properties for isomer 6a (Figure 2): ${ }^{1} \mathrm{H}-\mathrm{NMR}\left(\mathrm{CDCl}_{3}\right.$, $400 \mathrm{MHz}): \delta 1.47,1.48\left(\mathrm{~s}, 2 \times 3 \mathrm{H}, \mathrm{H}_{\mathrm{I}}\right), 3.87-3.91\left(\mathrm{~m}, 3 \mathrm{H}, \mathrm{H}_{\mathrm{B}}\right), 3.97-4.03\left(\mathrm{~m}, 3 \mathrm{H}, \mathrm{H}_{\mathrm{A}}\right.$ and $\left.\mathrm{H}_{\mathrm{E}}\right), 4.04$ $\left(\mathrm{dd}, J=12 \mathrm{~Hz}, 6 \mathrm{~Hz}, 1 \mathrm{H}, \mathrm{H}_{\mathrm{D} 2}\right), 4.07\left(\mathrm{dd}, J=8.4 \mathrm{~Hz}, 6.8 \mathrm{~Hz}, 1 \mathrm{H}, \mathrm{H}_{\mathrm{A}}\right), 4.21-4.27\left(\mathrm{~m}, 2 \mathrm{H}, \mathrm{H}_{\mathrm{C}}\right.$ and $\left.\mathrm{H}_{\mathrm{D}}\right)$, 
$5.72\left(\mathrm{dd}, J=8.8 \mathrm{~Hz}, 4 \mathrm{~Hz}, \mathrm{H}_{\mathrm{F}}\right), 5.99$ (br.s, 2H, NH ), $8.11\left(\mathrm{~s}, 1 \mathrm{H}, \mathrm{H}_{\mathrm{G}}\right), 8.54\left(\mathrm{~s}, 1 \mathrm{H}, \mathrm{H}_{\mathrm{H}}\right)$ ppm. ${ }^{13} \mathrm{C}-\mathrm{NMR}\left(\mathrm{CDCl}_{3}, 100 \mathrm{MHz}\right): \delta 25.2,26.4,66.1,68.5,60.4,74.1,82.0,110.3,111.1,144.0,151.3$, 154.0, 161.6 ppm. For the mixture of $\mathbf{6 a}$ and $\mathbf{6 b}$ : IR (neat): 3418, 3290, 3149, 2979, 2905, 1625, 1595, $1062 \mathrm{~cm}^{-1}$. HRMS (ESI): Calcd. for $\mathrm{C}_{14} \mathrm{H}_{19} \mathrm{~N}_{5} \mathrm{O}_{4}[\mathrm{M}+\mathrm{Na}]^{+}$322.1516, Found 322.1513.

Figure 2. Structure of compound $6 \mathbf{a}$.

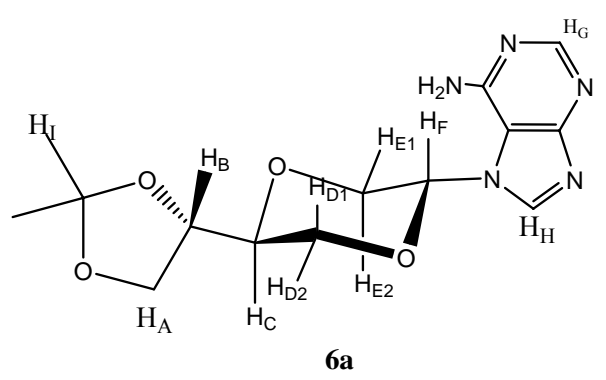

N-6-Benzoyl-N-9-\{(2R,5S)-5-[(4S)-(2,2-dimethyl-1,3-dioxolan-4-yl)]-1,4-dioxan-2-yl\} adenine (8a) and N-6-benzoyl-N-9-\{(2S,5S)-5-[(4S)-(2,2-dimethyl-1,3-dioxolan-4-yl)]-1,4-dioxan-2-yl\}-adenine (8b). N6-benzoyladenine $(2.065 \mathrm{~g}, 8.7 \mathrm{mmol})$ and ammonium sulfate $(120 \mathrm{mg}, 0.9 \mathrm{mmol})$ in HMDS $(50 \mathrm{~mL})$ was refluxed overnight. The solution was cooled and concentrated under reduced pressure. The residue was dissolved in dry acetonitrile $(20 \mathrm{~mL})$ and added a solution of $6(0.77 \mathrm{~g}, 3.1 \mathrm{mmol})$ in dry acetonitrile $(5 \mathrm{~mL})$. The mixture was then cooled to $0{ }^{\circ} \mathrm{C}$ and added TMSOTf $(0.6 \mathrm{~mL}, 3.3 \mathrm{mmol})$. The mixture was stirred for four hours. Then chloroform $(50 \mathrm{~mL})$ was added and the solution washed twice with saturated $\mathrm{NaHCO}_{3}$ solution $(20 \mathrm{~mL})$. The organic phase was dried over anhydrous $\mathrm{MgSO}_{4}$ filtered and concentrated under reduced pressure. The crude product was purified by flash chromatography using a gradient eluent system, first diethyl ether, followed by a mixture of dichloromethane and methanol (19:1) to afford the product as a yellow solid $(0.55 \mathrm{~g}, 42 \%)$ as a $3: 1$ mixture of trans- and cis products 8a and 8b. Pure 8a was obtained by repeated chromatography. Product 8a (Figure 3) exhibited the following spectroscopic properties: ${ }^{1} \mathrm{H}-\mathrm{NMR}\left(\mathrm{CDCl}_{3}, 400 \mathrm{MHz}\right)$ : $\delta 1.41,1.52\left(\mathrm{~s}, 2 \times 3 \mathrm{H}, \mathrm{H}_{\mathrm{G}}\right), 3.48\left(\mathrm{dd}, J=11.4 \mathrm{~Hz}, 9.4 \mathrm{~Hz}, 1 \mathrm{H}, \mathrm{H}_{\mathrm{E} 2}\right), 3.79-3.84\left(\mathrm{~m}, 1 \mathrm{H}, \mathrm{H}_{\mathrm{C}}\right), 3.94-3.97$ $\left(\mathrm{m}, 1 \mathrm{H}, \mathrm{H}_{\mathrm{A}}\right), 4.00\left(\mathrm{dd}, J=11.6 \mathrm{~Hz}, 10.8 \mathrm{~Hz}, 1 \mathrm{H}, \mathrm{H}_{\mathrm{D} 1}\right), 4.09\left(\mathrm{dd}, J=8.4 \mathrm{~Hz}, 6.8 \mathrm{~Hz}, 1 \mathrm{H}, \mathrm{H}_{\mathrm{A}}\right), 4.17(\mathrm{dd}$, $\left.J=11.6 \mathrm{~Hz}, 2.6 \mathrm{~Hz}, 1 \mathrm{H}, \mathrm{H}_{\mathrm{D} 2}\right), 4.20-4.25\left(\mathrm{~m}, 1 \mathrm{H}, \mathrm{H}_{\mathrm{B}}\right), 4.54\left(\mathrm{dd}, J=11.4 \mathrm{~Hz}, 2.6 \mathrm{~Hz}, \mathrm{H}_{\mathrm{E} 1}\right), 6.64(\mathrm{dd}$, $\left.J=9.2 \mathrm{~Hz}, 2.8 \mathrm{~Hz}, 1 \mathrm{H}, \mathrm{H}_{\mathrm{F}}\right), 7.43-7.49\left(\mathrm{~m}, 2 \mathrm{H}, \mathrm{H}_{\mathrm{K}}\right), 7.50-7.54\left(\mathrm{~m}, 1 \mathrm{H}, \mathrm{H}_{\mathrm{L}}\right), 8.23-8.28\left(\mathrm{~m}, 2 \mathrm{H}, \mathrm{H}_{\mathrm{J}}\right)$ ppm. ${ }^{13} \mathrm{C}-\mathrm{NMR}\left(\mathrm{CDCl}_{3}, 100 \mathrm{MHz}\right): \delta 25.4,26.4,62.8,65.0,66.6,74.1,74.4,81.9,110.3,115.2$, $128.4,130.1,132.4,137.5,142.1,144.9,149.3,157.2,175.6$ ppm. For the mixture of 8a and 8b: IR (neat): 3214, 2985, 1635, 1597, 1480, 1116, 1067, $1048 \mathrm{~cm}^{-1}$. HRMS (ESI): Calcd. for $\mathrm{C}_{21} \mathrm{H}_{23} \mathrm{~N}_{5} \mathrm{O}_{5}$ $[\mathrm{M}+\mathrm{H}]^{+}$426.1778, Found 426.1776.

Figure 3. Structure of compound 8a.

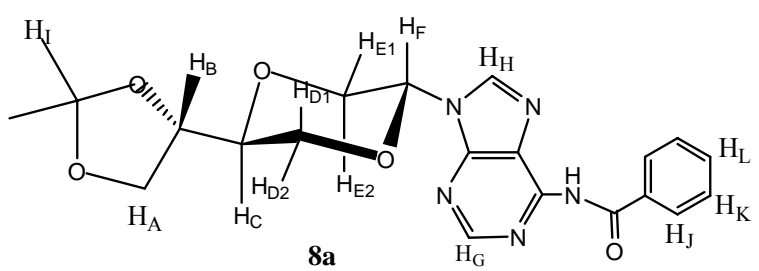


(2R,5S)-5-[(1S)-(1,2-Diacetyloxy)]-2-acetyloxy-1,4-dioxane (10a) and (2S,5S)-5-[(1S)-(1,2-diacetyloxy)]-2-acetyloxy-1,4-dioxane (10b). Compound $3(2.2 \mathrm{~g}, 10.8 \mathrm{mmol})$ and $p$-TsOH (2.26 g, $11.9 \mathrm{mmol}$ ) was dissolved in acetic anhydride $(50 \mathrm{~mL})$. The mixture was stirred for 7 hours at room temperature and then poured into ice water. To the mixture was neutralized with sodium bicarbonate, and then extracted with ethyl acetate. The organic phase was dried over anhydrous $\mathrm{MgSO}_{4}$ filtration and evaporated under reduced pressure. The residue was purified by flash chromatography using a mixture of diethyl ether and $n$-hexane (2:1) as the eluent. The product appeared as yellow oil, (2.35 g, $75 \%$ ). The diastereomers were not separated, however the NMR of the one diastereomer (Figure 4) was assigned the NMR signals: ${ }^{1} \mathrm{H}-\mathrm{NMR}\left(\mathrm{CDCl}_{3}, 400 \mathrm{MHz}\right): \delta 2.07,2.14,2.15\left(\mathrm{~s}, 3 \times 3 \mathrm{H}, \mathrm{CH}_{3}\right)$, 3.59-3.61(m, $\left.1 \mathrm{H}, \mathrm{H}_{\mathrm{D}}\right), 3.83\left(\mathrm{dd}, J=12.8 \mathrm{~Hz}, 2 \mathrm{H}, 1 \mathrm{H}, \mathrm{H}_{\mathrm{E}}\right), 3.89-3.98\left(\mathrm{~m}, 3 \mathrm{H}, \mathrm{H}_{\mathrm{E}}, \mathrm{H}_{\mathrm{D}}\right.$ and $\left.\mathrm{H}_{\mathrm{C}}\right), 4.16$ $\left(\mathrm{dd}, J=12 \mathrm{~Hz}, 6.8 \mathrm{~Hz}, 1 \mathrm{H}, \mathrm{H}_{\mathrm{A}}\right), 4.36\left(\mathrm{dd}, J=11.8 \mathrm{~Hz}, 4.2 \mathrm{~Hz}, 1 \mathrm{H}, \mathrm{H}_{\mathrm{A}}\right), 5.15-5.18\left(\mathrm{~m}, 1 \mathrm{H}, \mathrm{H}_{\mathrm{B}}\right), 5.87$ (d, 2Hz, 1H, $\left.\mathrm{H}_{\mathrm{F}}\right)$ ppm. ${ }^{13} \mathrm{C}-\mathrm{NMR}\left(\mathrm{CDCl}_{3}, 100 \mathrm{MHz}\right): \delta 20.7,20.8,21.1,61.2,62.1,68.1,69.8,73.6$, $88.2,169.8,170.2,170.5 \mathrm{ppm}$. The NMR data for the other diastereomer were the following: ${ }^{1} \mathrm{H}-\mathrm{NMR}$ $\left(\mathrm{CDCl}_{3}, 400 \mathrm{MHz}\right): \delta 2.10,2.11,2.16\left(\mathrm{~s}, 3 \times 3 \mathrm{H}, \mathrm{CH}_{3}\right), 3.78-3.82\left(\mathrm{~m}, 1 \mathrm{H}, \mathrm{H}_{\mathrm{C}}\right), 3.83(\mathrm{dd}, 12.8 \mathrm{~Hz}, 2 \mathrm{~Hz}$, $\left.1 \mathrm{H}, \mathrm{H}_{\mathrm{E}}\right), 3.93\left(\mathrm{~d}, 12.8 \mathrm{~Hz}, 1 \mathrm{H}, \mathrm{H}_{\mathrm{E}}\right), 4.13-4.26\left(\mathrm{~m}, 5 \mathrm{H}, \mathrm{H}_{\mathrm{B}}, \mathrm{H}_{\mathrm{D}}\right.$ and $\left.\mathrm{H}_{\mathrm{A}}\right), 5.87\left(\mathrm{~d}, 2 \mathrm{~Hz}, 1 \mathrm{H}, \mathrm{H}_{\mathrm{F}}\right)$ ppm. ${ }^{13} \mathrm{C}-\mathrm{NMR}\left(\mathrm{CDCl}_{3}, 100 \mathrm{MHz}\right): \delta$ 20.7, 20.8, 21.4, 63.1, 63.4, 67.6, 68.8, 74.2, 89.0, 169.7, 170.5, 170.6 ppm. IR (neat): 2959, 1737, 1219, 1044, $1014 \mathrm{~cm}^{-1}$. MS (m/z): 289.3, 243.3, 231.3, 217.3.

Figure 4. Structure of compound 10.

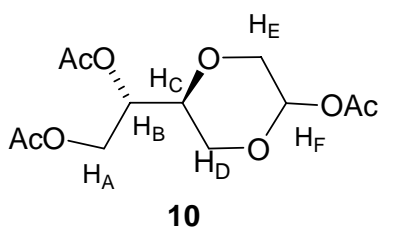

N-6-Benzoyl-N-9-\{(2R,5S)-5-[(1S)-(1,2-diacetyloxy)]-1,4-dioxan-2-yl\}adenine (11a) and N-6-benzoyl$N-9-\{(2 S, 5 S)-5-[(1 S)-(1,2$-diacetyloxy)]-1,4-dioxan-2-yl\}adenine (11b). N-6-benzoyladenine (1.09 g, $4.6 \mathrm{mmol})$ and ammonium sulfate $(62 \mathrm{mg}, 0.47 \mathrm{mmol})$ in HMDS $(50 \mathrm{~mL})$ was refluxed overnight. The mixture containing the silylated $N$-6-benzoyladenine 7 was concentrated under reduced pressure, dissolved in dry dichloroethane $(24 \mathrm{~mL})$ and then added a solution of $\mathbf{1 0}(0.74 \mathrm{~g}, 2.6 \mathrm{mmol})$ in dry dichloromethane $(6 \mathrm{~mL})$. This mixture was added TMSOTf $(0.94 \mathrm{~mL}, 5.2 \mathrm{mmol})$ at $0{ }^{\circ} \mathrm{C}$ and stirred overnight at room temperature. Chloroform $(50 \mathrm{~mL})$ was added to the mixture which was washed with saturated $\mathrm{NaHCO}_{3}$ solution $(25 \mathrm{~mL})$. The aqueous phase was extracted with chloroform $(50 \mathrm{~mL})$. The combined organic layers were dried over anhydrous $\mathrm{Na}_{2} \mathrm{SO}_{4}$ and filtered and concentrated under reduced pressure. The residue was purified by flash chromatography using a mixture of dichloromethane and methanol (9/1) as the eluent to afford the product $(0.67 \mathrm{~g}, 56 \%)$ as a mixture of the trans and cis products, 11a and 11b. The product was used for the next reaction without further purification. The NMR was too complex to allow reasonable assignments of the signals. IR (neat): 2958, 1737, 1636, 1216.1045, $1023 \mathrm{~cm}^{-1}$. HRMS (ESI): Calcd. for $\mathrm{C}_{22} \mathrm{H}_{23} \mathrm{~N}_{5} \mathrm{O}_{7}[\mathrm{M}+\mathrm{H}]^{+} 470.1677$, Found 470.1663. 
N-6-Benzoyl-N-9-\{(2R,5S)-5-[(1S)-(1,2-dihydroxyl)]-1,4-dioxan-2-yl\}adenine (9a) and N-6-benzoyl-N9-\{(2S,5S)-5-[(1S)-(1,2-dihydroxyl)]-1,4-dioxan-2-yl\}adenine (9b). Compound 11 (1.27 g, $30 \mathrm{~mL})$ was dissolved in $7 \mathrm{~N}$ ammonia in methanol $(30 \mathrm{~mL})$ and stirred for 7 hours at room temperature. The solution was concentrated and purified by flash chromatography using a mixture of dichloromethane and methanol (19/1) as the eluent. The pure trans- product 9a was isolated (140 mg, 13.5\%, Figure 5). Product 9a exhibited the following spectroscopic properties: ${ }^{1} \mathrm{H}-\mathrm{NMR}\left(\mathrm{CD}_{3} \mathrm{OD}, 400 \mathrm{MHz}\right): \delta 3.59$ (dd, $\left.J=11.2 \mathrm{~Hz}, 9.4 \mathrm{~Hz}, 1 \mathrm{H}, \mathrm{H}_{\mathrm{E} 2}\right), 3.60-3.70\left(\mathrm{~m}, 3 \mathrm{H}, \mathrm{H}_{\mathrm{A}}\right.$ and $\left.\mathrm{H}_{\mathrm{B}}\right), 3.89\left(\mathrm{dt}, J=10.6 \mathrm{~Hz}, 2.8 \mathrm{~Hz}, 1 \mathrm{H}, \mathrm{H}_{\mathrm{C}}\right)$, $4.13\left(\mathrm{dd}, J=11.8,10.6 \mathrm{~Hz}, 1 \mathrm{H}, \mathrm{H}_{\mathrm{D} 1}\right), 4.24\left(\mathrm{dd}, J=11.8,2.8 \mathrm{~Hz}, 1 \mathrm{H}, \mathrm{H}_{\mathrm{D} 2}\right), 4.37$ (dd, $J=11.2 \mathrm{~Hz}$, $\left.2.6 \mathrm{~Hz}, 1 \mathrm{H}, \mathrm{H}_{\mathrm{E} 1}\right), 6.55\left(\mathrm{dd}, J=9.4,2.6 \mathrm{~Hz}, 1 \mathrm{H}, \mathrm{H}_{\mathrm{F}}\right), 7.41-7.45\left(\mathrm{~m}, 1 \mathrm{H}, \mathrm{H}_{\mathrm{I}}\right), 7.49-7.53\left(\mathrm{~m}, 2 \mathrm{H}, \mathrm{H}_{\mathrm{K}}\right)$, 8.21-8.24 (m, 1H, $\left.\mathrm{H}_{\mathrm{C}}\right), 8.24\left(\mathrm{~s}, 1 \mathrm{H}, \mathrm{H}_{\mathrm{G}}\right), 8.67\left(\mathrm{~s}, 1 \mathrm{H}, \mathrm{H}_{\mathrm{H}}\right) \mathrm{ppm} .{ }^{13} \mathrm{C}-\mathrm{NMR}\left(\mathrm{CD}_{3} \mathrm{OD}, 100 \mathrm{MHz}\right): \delta 63.8$, 70.4, 70.8, 72.1, 76.3, 82.6, 115.5, 129.3, 131.0, 133.4, 138.2, 144.2, 145.2, 149.7, 156.9, 176.6ppm. IR (neat): 3286, 2876, 1635, 1424, 1285, 1115, 1063, 1021 $\mathrm{cm}^{-1}$. HRMS (ESI): Calcd. for $\mathrm{C}_{18} \mathrm{H}_{19} \mathrm{~N}_{5} \mathrm{O}_{5}$ $[\mathrm{M}+\mathrm{H}]^{+} 470.1677$, Found 470.1668 .

Figure 5. Structure of compound 9a.

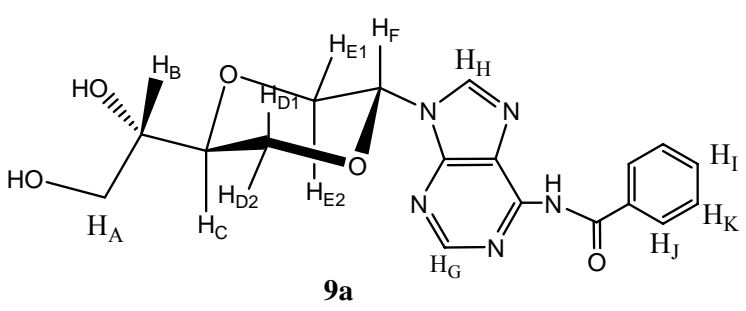

N-6-Benzoyl-N-9-\{(2S,5S)-5-[(1S)-hydroxyl-2-O-(4,4-dimethoxytrityl)-ethyl-1-yl]-1,4-dioxan-2-yl\}adenine (12). Compound 9 (mixture of 9a and 9b, $139 \mathrm{mg}, 0.36 \mathrm{mmol}$ ) and dimethoxyltrityl chloride (277 $\mathrm{mg}, 0.79 \mathrm{mmol})$ were dissolved in dry pyridine $(10 \mathrm{~mL})$. The solution was stirred at room temperature for 3 hours. The solution was concentrated in vacuo and residue was purified by flash chromatography using first diethyl ether and then ethyl acetate as the eluent to provide the product (12a and 12b) as a white solid (100 mg, 40\%). Product 12a (Figure 6) was obtained upon repeated chromatography and exhibited the following spectroscopic properties: ${ }^{1} \mathrm{H}-\mathrm{NMR}\left(\mathrm{CDCl}_{3}, 400 \mathrm{MHz}\right): \delta$ $2.82(\mathrm{~d}, J=4.8 \mathrm{~Hz}, 1 \mathrm{H}, \mathrm{OH}), 3.25\left(\mathrm{dd}, J=9.6 \mathrm{~Hz}, 5.4 \mathrm{~Hz}, 1 \mathrm{H}, \mathrm{H}_{\mathrm{A}}\right), 3.34\left(\mathrm{dd}, J=9.6 \mathrm{~Hz}, 5.6 \mathrm{~Hz}, \mathrm{H}_{\mathrm{A}}\right)$, $3.43\left(\mathrm{dd}, J=11.2 \mathrm{~Hz}, 9.2 \mathrm{~Hz}, \mathrm{H}_{\mathrm{E} 2}\right), 3.75-3.82\left(\mathrm{~m}, 1 \mathrm{H}, \mathrm{H}_{\mathrm{B}}\right), 3.77\left(\mathrm{~s}, 2 \times 3 \mathrm{H}, \mathrm{OCH}_{3}\right), 3.86-3.90(\mathrm{~m}, 1 \mathrm{H}$, $\left.\mathrm{H}_{\mathrm{C}}\right), 4.00\left(\mathrm{dd}, J=12 \mathrm{~Hz}, 10.2 \mathrm{~Hz}, \mathrm{H}_{\mathrm{D} 1}\right), 4.13\left(\mathrm{dd}, J=12 \mathrm{~Hz}, 2.8 \mathrm{~Hz}, \mathrm{H}_{\mathrm{D} 2}\right), 4.43$ (dd, $J=11.2 \mathrm{~Hz}$, $\left.2.8 \mathrm{~Hz}, \mathrm{H}_{\mathrm{E}}\right), 6.60$ (dd, $\left.J=9.2 \mathrm{~Hz}, 2.8 \mathrm{~Hz}, \mathrm{H}_{\mathrm{F}}\right), 6.82-6.86(\mathrm{~m}, 5 \mathrm{H}$, aromatic protons), 7.20-7.24 (m, 1H, aromatic proton), 7.26-7.37 (m, 5H, aromatic protons), 7.40-7.53 (m, 5H, aromatic protons), 8.13 (s, $\left.1 \mathrm{H}, \mathrm{H}_{\mathrm{G}}\right), 8.15-8.25\left(\mathrm{~m}, 2 \mathrm{H}\right.$, aromatic protons), $8.64\left(\mathrm{~s}, 1 \mathrm{H}, \mathrm{H}_{\mathrm{H}}\right) \mathrm{ppm} .{ }^{13} \mathrm{C}-\mathrm{NMR}\left(\mathrm{CDCl}_{3}, 100 \mathrm{MHz}\right): \delta$ $55.2,63.8,69.3,69.4,70.0,75.5,81.0,86.5,113.2,126.9,129.8,130.0,132.3,135.7,135.8,137.1$, 142.1, 143.0, 144.6, 148.4, 157.1, 158.6, 175.4 ppm. HRMS (ESI): Calcd. for $\mathrm{C}_{39} \mathrm{H}_{37} \mathrm{~N}_{5} \mathrm{O}_{7}[\mathrm{M}+\mathrm{Na}]^{+}$ 710.2591 , Found 710.2588 . 
Figure 6. Structure of compound 12a.

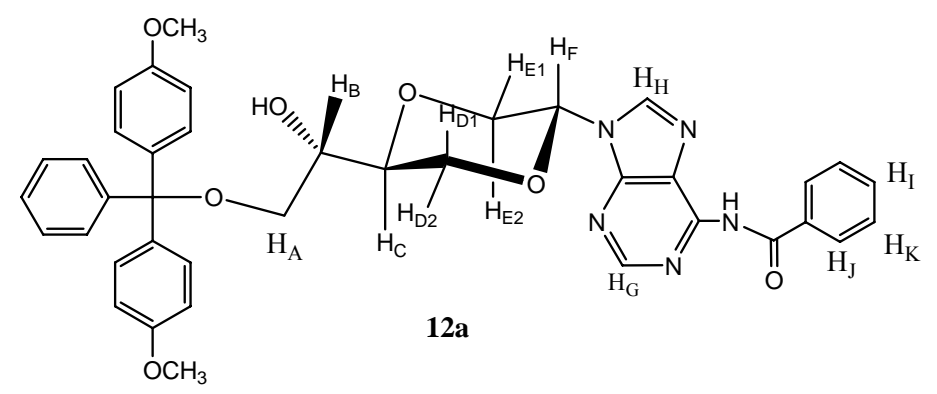

N-6-Benzoyl-N-9-\{(2S, 5S)-5-\{(1S)-O-[2-cyanoethoxy(diisopropylamino)phosphino]-2-O-(4,4'-dimethoxytrityl)-ethyl-1-yl\}-1,4-dioxan-2-yl;adenine (13a). Compound 12 (mixture of 12a and 12b) (99 $\mathrm{mg}, 0.14 \mathrm{mmol})$ was dissolved in dry dicholormethane $(6 \mathrm{~mL})$ was then added $N, N$-diisopropylethylamine $(50 \mu \mathrm{L}, 0.29 \mathrm{mmol})$. Then $N, N$-diisopropyl-2-cyanoethyl phosphoramidic chloride (105 $\mu \mathrm{L}, 0.47 \mathrm{mmol}$ ) was added. The resulting solution was stirred for 6 hours at room temperature. The solution was then concentrated and purified by flash chromatography using a mixture of ethyl acetate and n-hexane (4:1) as the eluent to give the trans isomer 13a (65 mg, 51\%) as a mixture of diastereomers due to the stereogenic phosphorus. Major diastereomer 13a (Figure 7) had the following spectroscopic properties: ${ }^{1} \mathrm{H}-\mathrm{NMR}\left(\mathrm{CDCl}_{3}, 400 \mathrm{MHz}\right): \delta 1.14\left(\mathrm{~d}, J=7.2 \mathrm{~Hz}, 2 \times 3 \mathrm{H}, \mathrm{H}_{\mathrm{K}}\right), 1.19(\mathrm{~d}$, $\left.J=7.2 \mathrm{~Hz}, 2 \times 3 \mathrm{H}, \mathrm{H}_{\mathrm{K}}\right), 2.64\left(\mathrm{dt}, J=6.4,2.4 \mathrm{~Hz}, 2 \mathrm{H}, \mathrm{H}_{\mathrm{I}}\right), 3.27\left(\mathrm{dd}, J=9.4,5.2 \mathrm{~Hz}, 1 \mathrm{H}, \mathrm{H}_{\mathrm{A}}\right), 3.36(\mathrm{dd}$, $\left.J=9.4,6 \mathrm{~Hz}, 1 \mathrm{H}, \mathrm{H}_{\mathrm{A}}\right), 3.45\left(\mathrm{dd}, J=11.2,9.4 \mathrm{~Hz}, 1 \mathrm{H}, \mathrm{H}_{\mathrm{E} 2}\right), 3.56-3.70\left(\mathrm{~m}, 2 \times 1 \mathrm{H}, \mathrm{H}_{\mathrm{L}}\right), 3.771,3.768$ $\left(\mathrm{s}, 2 \times 3 \mathrm{H}, \mathrm{OCH}_{3}\right), 3.72-3.82\left(\mathrm{~m}, 1 \mathrm{H}, \mathrm{H}_{\mathrm{J}}\right), 3.87-3.95\left(\mathrm{~m}, 1 \mathrm{H}, \mathrm{H}_{\mathrm{J}}\right), 4.00-4.09\left(\mathrm{~m}, 3 \times 1 \mathrm{H}, \mathrm{H}_{\mathrm{B}}, \mathrm{H}_{\mathrm{D}}, \mathrm{H}_{\mathrm{C}}\right)$, $4.24\left(\mathrm{~d}, J=10 \mathrm{~Hz}, 1 \mathrm{H}, \mathrm{H}_{\mathrm{D}}\right), 4.40\left(\mathrm{dd}, J=11.2,2.6 \mathrm{~Hz}, 1 \mathrm{H}, \mathrm{H}_{\mathrm{E} 1}\right), 6.61\left(\mathrm{dd}, J=9.4,2.6 \mathrm{~Hz}, 1 \mathrm{H}, \mathrm{H}_{\mathrm{F}}\right)$, 6.81-6.85 (m, 4H, aromatic H), 7.19-7.22 (m, 1H, aromatic H), 7.27-7.30 (m, 2H, aromatic H), 7.34-7.36 (m, 4H, aromatic $\mathrm{H}), 7.41-7.48(\mathrm{~m}, 4 \mathrm{H}$, aromatic $\mathrm{H}), 7.52-7.56(\mathrm{~m}, 4 \mathrm{H}$, aromatic $\mathrm{H}), 8.14$ $\left(\mathrm{s}, 1 \mathrm{H}, \mathrm{H}_{\mathrm{G}}\right), 8.22-8.25(\mathrm{~m}, 2 \mathrm{H}$, aromatic $\mathrm{H}), 8.69\left(\mathrm{~s}, 1 \mathrm{H}, \mathrm{H}_{\mathrm{H}}\right), 12.59(\mathrm{~s}, 1 \mathrm{H}, \mathrm{NH}) \mathrm{ppm} .{ }^{13} \mathrm{C}-\mathrm{NMR}$ $\left(\mathrm{CDCl}_{3}, 100 \mathrm{MHz}\right): \delta 20.4,20.5,24.56,24.64,24.67,24.74,43.2,43.4,55.2,58.0,58.2,60.4$,69.55, 69.62, 72.1, 75.3, 75.4, 81.3, 86.4, 113.1, 114.4, 117.7, 126.8, 127.8, 128.2, 128.3, 129.9, 130.05, 130.09, 132.3, 135.95, 136.01, 137.2, 141.8, 143.3, 144.8, 148.4, 157.2, 158.5, 175.5 ppm. ${ }^{31} \mathrm{P}-\mathrm{NMR}$ $\left(\mathrm{CDCl}_{3}\right): 151.0,152.0$ (small) ppm. IR (neat): 3239, 3056, 2965, 2929, 2362, 2338, 1637, 1507, 1251, 1083, 788, 754, $719 \mathrm{~cm}^{-1}$. HRMS (ESI): Calcd. for $\mathrm{C}_{48} \mathrm{H}_{54} \mathrm{~N}_{7} \mathrm{O}_{8} \mathrm{P}[\mathrm{M}+\mathrm{Na}]^{+}$910.3669, Found 910.3639.

Figure 7. Structure of compound 13a.

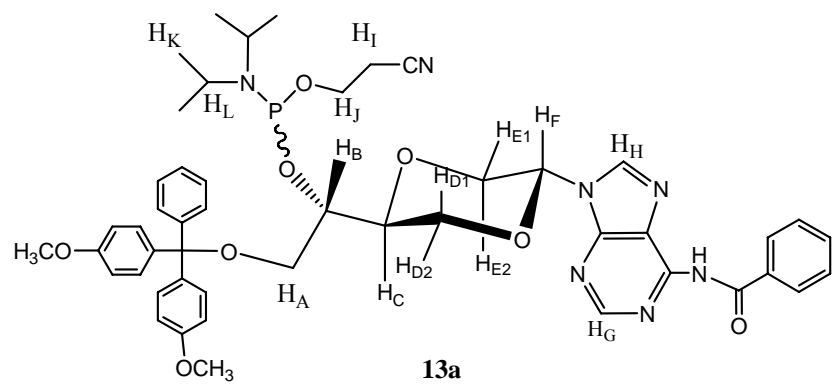


1-[(2R,5S)-5-[(1S)-Acetyloxy-2-hydroxylethyl-1-yl]-1,4-dioxan-2-yl]uracil (14a). The synthesis of this compound has been reported earlier [7]. Compound 14a (Figure 8) exhibited the following spectroscopic properties: ${ }^{1} \mathrm{H}-\mathrm{NMR}\left(\mathrm{CD}_{3} \mathrm{OD}, 400 \mathrm{MHz}\right): \delta 2.07\left(\mathrm{~s}, 3 \mathrm{H}, \mathrm{CH}_{3}\right), 3.58(\mathrm{dd}, J=11.4 \mathrm{~Hz}$, $\left.9.8 \mathrm{~Hz}, 1 \mathrm{H}, \mathrm{H}_{\mathrm{E} 2}\right), 3.70-3.74\left(\mathrm{~m}, 1 \mathrm{H}, \mathrm{H}_{\mathrm{B}}\right), 3.77-3.82\left(\mathrm{~m}, 1 \mathrm{H}, \mathrm{H}_{\mathrm{C}}\right), 3.92-3.98\left(\mathrm{~m}, 2 \mathrm{H}, \mathrm{H}_{\mathrm{E}}\right.$ and $\left.\mathrm{H}_{\mathrm{D} 1}\right), 4.06$ $\left(\mathrm{dd}, J=11.6,2.8 \mathrm{~Hz}, 1 \mathrm{H}, \mathrm{H}_{\mathrm{D} 2}\right), 4.13\left(\mathrm{dd}, J=11.2,6.4 \mathrm{~Hz}, 1 \mathrm{H}, \mathrm{H}_{\mathrm{A}}\right), 4.16(\mathrm{dd}, J=11.2 \mathrm{~Hz}, 5.4 \mathrm{~Hz}, 1 \mathrm{H}$, $\left.\mathrm{H}_{\mathrm{A}}\right), 5.69\left(\mathrm{dd}, J=10 \mathrm{~Hz}, 2.8 \mathrm{~Hz}, 1 \mathrm{H}, \mathrm{H}_{\mathrm{F}}\right), 5.70\left(\mathrm{~d}, J=8 \mathrm{~Hz}, 1 \mathrm{H}, \mathrm{H}_{\mathrm{H}}\right), 7.71\left(\mathrm{~d}, J=8 \mathrm{~Hz}, 1 \mathrm{H}, \mathrm{H}_{\mathrm{G}}\right) \mathrm{ppm}$. ${ }^{13} \mathrm{C}-\mathrm{NMR}\left(\mathrm{CD}_{3} \mathrm{OD}, 100 \mathrm{MHz}\right): \delta 20.9,66.4,69.2,69.4,69.8,76.0,80.0,103.1,142.3,151.9,166.0$, 172.8 ppm. IR (neat): 3477, 3190, 3110, 3074, 2996, 2879, 1697, 1268, $1105 \mathrm{~cm}^{-1}$. MS (EI): 230.3, 197( $\left.\mathrm{M}^{+}-\left(\mathrm{CH}_{3} \mathrm{COCHCH}_{2} \mathrm{OH}\right)\right), 189\left(\mathrm{M}^{+}\right.$-uracil). HRMS (ESI): Calcd. for $\mathrm{C}_{12} \mathrm{H}_{16} \mathrm{~N}_{2} \mathrm{O}_{7}[\mathrm{M}+\mathrm{Na}]^{+}$ 323.0856, Found 323.0862.

Figure 8. Structure of compound 14.

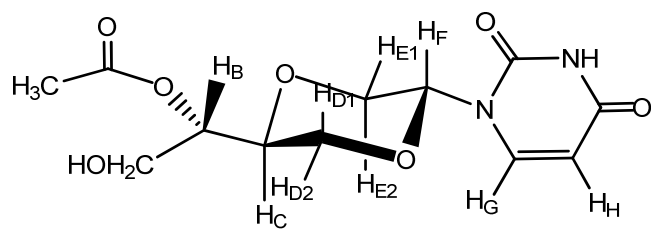

14

N6-Benzoyladenine uracil dinucleotide (15). Compound 13a (30 mg, $0.039 \mathrm{mmol})$ and 14a (8 mg, $0.027 \mathrm{mmol}$ ) in a reaction flask with a magnetic stirring bar were dried under high vacuum for 4 hours. Then a mixture $1-H$ tetrazole $(0.3 \mathrm{~mL}, 0.45 \mathrm{M}, 0.135 \mathrm{mmol})$ in dry acetonitrile $(6 \mathrm{~mL})$ was added under a nitrogen atmosphere. The solution was stirred overnight at room temperature. Then was added a few drops of iodine in THF (1M solution), 2,6-lutidine and $\mathrm{H}_{2} \mathrm{O}(2: 2: 1)$ until an orange color persisted. The solution was then quenched with saturated sodium thiosulfate solution $(4 \mathrm{~mL})$. Then the two phases were treated with saturated $\mathrm{NaHCO}_{3}$ solution $(4 \mathrm{~mL})$. The separated aqueous phase was extracted with $4 \times 8 \mathrm{~mL}$ of dichloromethane. The combined organic phase was dried over anhydrous $\mathrm{Na}_{2} \mathrm{SO}_{4}$ and filtered and the solvent evaporated. Flash chromatographic purification, using mixtures of dichloromethane and methanol in the ratio from 25:1 to 15:1 as gradient solvents, afforded a product (16 mg, 61.5\%), which was assigned structure 15 (Figure 9) based on the following spectroscopic properties: ${ }^{1} \mathrm{H}-\mathrm{NMR}\left(\mathrm{CDCl}_{3}, 400 \mathrm{MHz}\right.$ ): $\delta 2.02$ (s, 3H, $\underline{\mathrm{C}}_{3} \mathrm{COO}$ ), 2.09 (s, 3H, $\mathrm{CH}_{3} \mathrm{COO}$ ), 2.51-2.62 (m, $\left.2 \mathrm{H}, \underline{\mathrm{C}}_{2} \mathrm{CN}\right), 2.71-2.74\left(\mathrm{~m}, 2 \mathrm{H}, \mathrm{C}_{2} \mathrm{CN}\right), 3.15(\mathrm{dd}, 1 \mathrm{H}, J=11.2 \mathrm{~Hz}, 9.6 \mathrm{~Hz}), 3.21-3.28(\mathrm{~m}), 3.38$ $(\mathrm{dd}, J=11.4 \mathrm{~Hz}, 9.8 \mathrm{~Hz}), 3.45-3.58(\mathrm{~m}), 3.62-3.96(\mathrm{~m}), 4.01(\mathrm{dd}, 1 \mathrm{H}, J=11.2 \mathrm{~Hz}, 2.8 \mathrm{~Hz}), 4.03-4.13$ (m), 4.14-4.35 (m), 4.37-4.47 (m), 4.63-4.74 (m), 5.58 (dd, 1H, $J=9.8 \mathrm{~Hz}, 3 \mathrm{~Hz}$, anomeric proton), 5.66-5.71 (m, 3H, two protons in uracil and one anomeric proton), 6.62-6.68 (m), 6.84-6.89 (m, aromatic protons), $7.20(\mathrm{~d}, J=8 \mathrm{~Hz}, 1 \mathrm{H}$, a proton in uracil), 7.22-7.28 (m, aromatic protons), 7.30-7.37 ( $\mathrm{m}$, aromatic protons and one proton in uracil), 7.42-7.49 (m, aromatic protons), 7.52-7.58 (m, aromatic protons), 8.143 (s, 1H, a proton in adenine), 8.148 (s, 1H, a proton in adenine), 8.23-8.25 ( $\mathrm{m}$, aromatic protons), 8.33 (br. $1 \mathrm{H}, \mathrm{NH}), 8.63$ (s, a proton in adenine), 8.64 (s, a proton in adenine) ppm. ${ }^{13} \mathrm{C}-\mathrm{NMR}\left(\mathrm{CDCl}_{3}, 100 \mathrm{MHz}\right): \delta$ 20.76, 20.82, 55.3, 62.0-62.5 (m), 67.6, 68.1, 68.4, 69.4, 73.2, $74.4,74.8,78.4,78.5,80.9,86.8,86.9,102.7,102.8,128.06,128.10,128.3,129.9,130.0,132.4$, $134.98,135.02,135.2,137.1,139.2,139.3,141.9,142.9,148.4,149.5,157.2,158.8,162.1,170.4$, 
175.6 ppm. ${ }^{31} \mathrm{P}-\mathrm{NMR}\left(\mathrm{CDCl}_{3}\right):-1.28,-1.49$ ppm. HRMS (ESI): Calcd. for $\mathrm{C}_{54} \mathrm{H}_{55} \mathrm{~N}_{8} \mathrm{O}_{16} \mathrm{P}[\mathrm{M}+\mathrm{Na}]^{+}$ 1125.3372, Found 1125.3354.

Figure 9. Structure of compound 15.

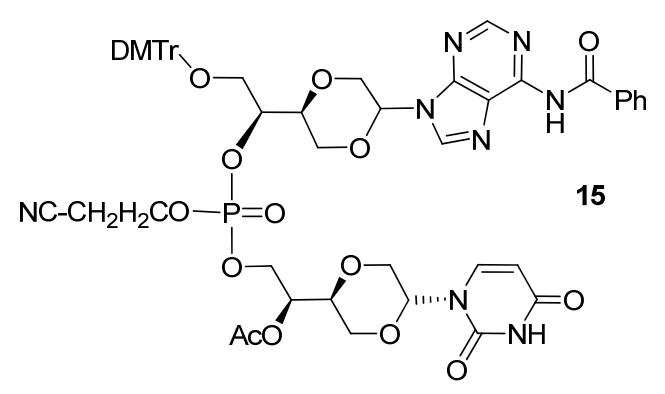

\section{Conclusions}

The synthesis of the adenosine analogs containing an optically active 1,4-dioxane sugar equivalent was achieved by coupling of $N$-silylated $N$-6-benzoyl protected adenine with the sugar acetate equivalents. Applying conventional phosphorimidite methodology the adenine analog was further coupled to the related uridine analog to give the corresponding protected dinucleotide, thus making it feasible that larger oligonucleotides can be prepared, including the application of conventional automated procedures.

\section{Acknowledgments}

The authors wish to thank the Norwegian Research Council, NFR, for financial support.

\section{References and Notes}

1. Ichikawa, E.; Kato, K. Sugar-modified nucleosides in past 10 years, a review. Curr. Med. Chem. 2001, 8, 385-423.

2. Szarek, W.A.; Pinto, B.M.; Iwakawa, M. Synthesis and biological activity of nucleoside analogues involving modifications in the carbohydrate ring. Can. J. Chem. 1985, 63, 2149-2161.

3. Hronowski, L.J.; Szarek, W.A. Synthesis and biological evaluation of novel pyrimidine nucleoside analogues of 1,4-oxathiane, 1,4-dithiane, and 1,4-dioxane. J. Med. Chem. 1982, 25, 522-526.

4. Prisbe, E.J. Synthesis and antiviral evaluation of 1,4-dioxane nucleoside analogues related to nucleoside dialdehydes. J. Med. Chem. 1986, 29, 2445-2450.

5. Mitsuaki, M.; Naoko, K.; Yamaizumi, Z.; Okamoto, Y.; Nagahara, K.; Takayanagi, H. Synthesis of a novel dioxane nucleoside having two bases, 2(R)-(5-fluorouracil-1-yl)-5(R)-hydroxymethyl3(R)-(uracil-1-yl)-1,4-dioxane and its 2(S)-isomer, from uridine. Tetrahedron Lett. 1997, 38, 6841-6844.

6. Szarek, W.A.; Vyas, D.M.; Achmatowicz, B. Synthesis of nucleoside analogues of 1,4-oxathiane, 1,4-dothiane, and 1,4-dioxane. J. Heteroc. Chem. 1975, 12, 123-127. 
7. Qiang, Y.; Carlsen, P. Synthesis of a Novel, Optically Active Uridine Analog Containing a 1,4dioxane Sugar Moiety. Synthesis of the Corresponding Dinucleotide. Nucleos. Nucleot. Nucl. Acids 2009, 28, 165-174.

8. Sinha, N.D.; Biernat, J.; Mcmanus, J. Köster, H. Polymer support oligonucleotide synthesis. XVIII: use of -cyanoethyl-N,N-dialkylamino-/N-morpholino phosphorami-dite of deoxynucleosides for the synthesis of DNA fragments simplifying deprotection and isolation of the final product. Nucl. Acids Res. 1984, 12, 4539-4557.

9. Nagashima, N.; Ohno, M. Selective monoalkylation of acyclic diols by means of dibutyltin oxide and fluoride salts. Chem. Pharm. Bull. 1991, 39, 1972-1982.

10. Nagashima, N.; Ohno, M. An efficient O-monoalkylation of dimethyl L-tartrate via O-stannylene acetal with alkyl halides in the presence of caesium fluoride. Chem. Lett. 1987, 1, 141-144.

11. Baskaran, S.; Aurich, H.G.; Biesemeier, F.; Harms, K. Cycloadducts from highly functionalized nitrones and oximes as ligands in the enantioselective addition of diethylzinc to benzaldehyde. J. Chem. Soc. Perkin Trans. 1 1998, 22, 3717-3724.

12. Marshall, J.A.; Andersen, N.H.; Hochstetler, A. Reduction of malonic enolates with lithium aluminum hydride. J. Org. Chem. 1967, 32, 113-118.

13. Shi, M.; Jiang, J.-K.; Feng, Y.-S. Chiral $C_{2}$-symmetric 2,3-disubstituted aziridine and 2,6disubstituted piperidine as chiral ligands in the addition reaction of diethylzinc with arylaldehydes. Tetrahedron Asymmetry 2000, 11, 4923-4933.

14. Marshall, J.A.; Beaudion, S. Stereoselective Synthesis of Higher Sugars by Homologation of Carbohydrate-Derived Enals with Nonracemic $\gamma$-(Silyloxy) Allylic Stannanes and SubstrateDirected Hydroxylation. J. Org. Chem. 1994, 59, 6614-6619.

15. Albizati, K.F.; Babu, S. A synthesis of the HIV-protease inhibitor nelfinavir from D-tartaric acid. Tetrahedron Lett. 2001, 42, 6481-6485.

16. Takano, S.; Kurotaki, A.; Sekiguchi, Y.; Satoh, S.; Hirama, M.; Ogasawara, K. Selective manipulation of hydroxy groups in (2S,3S)-threitol. Synthesis 1986, 10, 811-817.

17. Vorbrüggen, H.; Krolikiewicz, K. Nucleoside syntheses. XXII. Nucleoside synthesis with trimethylsilyl triflate and perchlorate as catalysts. Chem. Ber. 1981, 114, 1234-1255.

18. Vorbrüggen, H.; Höfle, G. Nucleoside syntheses. XXIII. On the mechanism of nucleoside synthesis. Chem. Ber. 1981, 114, 1256-1268.

19. Green, T.W.; Wuts, P.G.M. Protective Groups in Organic Synthesis; Wiley \& Sons: New York, NY, USA, 1999.

20. Magdalena, J.; Fernandez, S.; Ferrero, M.; Gotor, V. Difficulties in the deprotection of 1,2-ketals in nucleosides containing alkylidencarbazoyl groups. Nucleos. Nucleoti. Nucl. Acids 2002, 21, 55-64.

21. Van Cleve, J.W.; Rist, C.E. Ethylidene derivatives of D-erythrose. I. 2,3-O-Ethylidene- $\beta-D$ erythrofuranose. Carbohydr. Res. 1967, 4, 82-92.

22. Angyal, S.J.; Beveridge, R.J. Conformational analysis in carbohydrate chemistry. Part IV. Intramolecular acetal formation by primary versus secondary hydroxyl groups. Carbohydr. Res. 1978, 65, 229-234. 
23. Shing, T.K.M.; Wan, L.H. Facile Syntheses of Valiolamine and Its Diastereomers from (-)-Quinic Acid. Nucleophilic Substitution Reactions of 5-(Hydroxy-methyl)cyclohexane-1,2,3,4,5-pentol. J. Org. Chem. 1996, 61, 8468-8479.

24. Kim, K.S.; Song, Y.H.; Lee, B.H.; Hahn, C.S. Efficient and selective cleavage of acetals and ketals using ferric chloride adsorbed on silica gel. J. Org. Chem. 1986, 51, 404-406.

25. Szarek, W.A.; Zamojski, A. A new, facile method for cleavage of acetals and dithioacetals. Tetrahedron Lett. 1986, 27, 3827-3830.

26. Hall, D.M.; Lawler, T.E.; Childress, B.C. A practical synthesis of 1,2,3,6-tetra- $O$-acetyl- $\alpha$ - and $\beta$-D-glucopyranose, and their use to prepare trisaccharides. Carbohydr. Res. 1974, 38, 359-363.

27. Saito, Y.; Zevaco, T.A.; Agrofoglio, L.A. Chemical synthesis of ${ }^{13} \mathrm{C}$ labeled anti-HIV nucleosides as mass-internal standards. Tetrahedron 2002, 58, 9593-9603.

28. Cao, Y.; Okada, Y.; Yamada, H. Facile and regioselective preparation of partly $O$-benzylated D-glucopyranose acetates via acid-mediated simultaneous debenzylation-acetolysis. Carbohydr. Res. 2006, 341, 2219-2223.

29. Neilson, T.; Werstiuk, E.S. Oligoribonucleotide synthesis. II. Preparation of 2'-Otetrahydropyranol derivatives of adenosine and cytidine necessary for insertion in stepwise synthesis. Can. J. Chem. 1971, 49, 493-499.

30. Fisher, E.F.; Caruthers, M.H. Color coded triarylmethyl protecting groups useful for deoxypolynucleotide synthesis. Nucl. Acids Res. 1983, 11, 1589-1599.

31. Stütz, J.A.R.; Richert, C. Synthesis of a formamidine-protected 5'-amino-2',5'-dideoxy-guanosine phosphoramidite and preparation of 5'-acylamidooligonucleotides. Tetrahedron Lett. 2004, 45, 509-513.

32. Eisenfuhr, A.; Arora, P.S. A ribozyme with michaelase activity: synthesis of the substrate precursors. Bioorg. Med. Chem. 2003, 11, 235-249.

33. Ferris, J.P.; Peyser, J.R. Rapid and efficient syntheses of phosphorylated dinucleotides. Nucleos. Nucleot. 1994, 13, 1087-1111.

Sample Availability: Not available

(C) 2011 by the authors; licensee MDPI, Basel, Switzerland. This article is an open access article distributed under the terms and conditions of the Creative Commons Attribution license (http://creativecommons.org/licenses/by/3.0/). 